REVISTA INTERNACIONAL DE CIENCIAS DEL DEPORTE International Journal of Sport Science

Rev. int. cienc. deporte

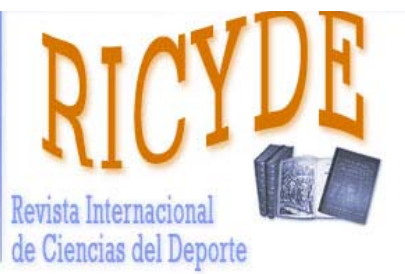

International Journal of Sport Science

VOLUMEN VI - AÑO VI

Páginas:196-204 ISSN:1885-3137

No 20 - Julio - 2010

\title{
Efecto de dos programas de actividad física en el medio acuático con diferente impacto, sobre el índice de rigidez óseo y el nivel de actividad física en mujeres postmenopáusicas y osteopénicas de Toledo
}

\section{Effects of two aquatic physical activity programs with different impact, on stiffness index and physical activity level in postmenopausal and osteopenic women from Toledo}

\author{
Germán Díaz Ureña \\ Universidad Europea de Madrid \\ María Carrasco Poyatos \\ Universidad de Murcia \\ Andrés Barriga Martín \\ Fernando Jiménez Díaz \\ Fernando Navarro Valdivielso \\ Universidad de Castilla la Mancha
}

\section{Resumen}

La osteoporosis es la enfermedad crónica más prevalente en el mundo, especialmente en mujeres mayores de 65 años. En España afecta a un $35 \%$ de las mujeres mayores de 50 años. Existen dos componentes que afectan a la fortaleza del hueso: cantidad de hueso, medido como densidad de masa ósea (DMO), y calidad ósea, que se puede medir como índice de rigidez óseo (SI). El ejercicio recomendado para la mejora de la DMO es fundamentalmente un ejercicio de impacto o de fuerza. El trabajo en el medio acuático para la mejora de la DMO presenta controversias sobre su posible utilidad. Lo mismo sucede con el índice de rigidez óseo (SI). Un total de 60 mujeres $(57,7+6,18$ años) fueron divididas en 2 grupos. Cada grupo realizó un entrenamiento en el medio acuático diferente: a) grupo de natación (GN), realizó trabajo en la piscina profunda; b) grupo de impacto y resistencia (GIR), realizó trabajo en la piscina poco profunda. El entrenamiento fue realizado durante 6 meses, 2 días por semana con una duración de 45 minutos por sesión. Se tomaron medidas del SI con el densitómetro de ultrasonidos portátil (Lunar Achilles Express). El nivel de actividad física fue medido mediante acelerómetros uniaxiales (MTI actigraph). Se tomaron medidas antropométricas de las participantes. Al comienzo del estudio no hubo diferencias entre ambos grupos en ninguna de las variables medidas. Tampoco hubo diferencias al finalizar los 6 meses de entrenamiento. En la única variable que hubo diferencias tras los 6 meses de entrenamiento fue en el peso del GIR, $(p<0,05)$. Un programa de entrenamiento de 6 meses de duración con dos sesiones semanales no es suficiente para la mejora del SI, ni para modificar los hábitos de actividad física de las participantes.

Palabras clave: osteoporosis; ejercicio acuático; mujeres.

\begin{abstract}
Osteoporosis is the most prevalent chronic illness in the world, specially between elderly women above 65 years old. In Spain, 35\% of elderly women above 50 years old suffer this illness. There are two components that affect bone strength: bone quantity, measured as bone mass density (BMD), and bone quality, which can be measured as bone stiffness index (SI). It is recommended impact and strength training to improve bone mineral density (BMD). Water-based exercise is unclear about the effect on BMD. The same goes for SI. Sixty elderly women ( $57,7+6,18$ years old) were divided into two groups: a) swimming group (GN), trained in deep water; b) impact and resistance group (GIR) trained in shallow water. The training was carried out during 6 months, 2 days per week during 45 minutes per session. BMD were measured by an ultrasound portable densitometer device (Lunar Achilles Express). Physical activity level was assessed with an uniaxial accelerometer (MTI actigraph). Antropometric measured were also assessed. There were no significance differences between groups in any of the measured variables neither at the beginning nor after six months of training. A significant decrease was observed on weight $(p<0,05)$ in GIR after the 6 -month protocol. A six months water-based exercise with two sessions per week seems to be not enough neither to increase BMD nor to change physical activity habits in postmenopausal women.
\end{abstract}

Key words: osteoporosis; water-based exercise; women.

Correspondencia/correspondence: Germán Díaz Ureña

Universidad Europea de Madrid. España

e-mail: German.diaz@uem.es 
Díaz, G.; Carrasco, M.; Barriga, A.; Jiménez, F.; Navarro, F. (2010). Efecto de dos programas de actividad física en el medio acuático con diferente impacto sobre el nivel de densidad ósea y el nivel de actividad física en mujeres postmenopáusicas y osteopénicas de Toledo. Revista Internacional de Ciencias del Deporte, 20(6), 196-204. http://www.cafyd.com/REVISTA/02002.pdf

\section{Introducción}

T a osteoporosis es la enfermedad crónica más prevalente en el mundo, especialmente en $\amalg$ mujeres mayores de 65 años (Valdivia y Szot, 1999). En España, afecta a un 35\% de las mujeres mayores de 50 años, ascendiendo a un 52\% en las mujeres mayores de 70 años (Cannata et al., 2005).

El principal problema de la osteoporosis es el aumento en el riesgo de fractura. En 1990, el número de fracturas por osteoporosis estimadas en Europa fue de 2,7 millones, que suponen un coste aproximado de 36 billones de euros, de las cuales dos terceras partes son fracturas de cadera (Kanis y Johnell, 2005). La Comisión Europea ha pronosticado un aumento del 135 \% de las fracturas de cadera en los próximos 50 años, con su aumento económico consecuente. En la actualidad se considera que un estilo de vida activo disminuye el riesgo de fracturas y por tanto actúa como un eficaz mecanismo preventivo (Astrom et al., 1987; Cooper et al., 1988; Coupland et al., 1999; Feskanich et al., 2002; Khan et al., 2000; Rutherford, 1999; Wickham et al., 1989). Para un aumento de la fortaleza del hueso, y por consiguiente reducción del riesgo de fractura, se pretende mejorar tanto la cantidad de hueso, medido como densidad mineral ósea (DMO), como la calidad, medida como el índice de rigidez óseo (SI).

El ejercicio recomendado para la mejora de la DMO es fundamentalmente un ejercicio de impacto, consistente en la aplicación de ejercicios de naturaleza pliométrica, como saltos, carrera, etc. (Asikainen et al., 2004; Bonaiuti et al., 2006; Drinkwater et al., 1995; Rutherford, 1999). Sin embargo, no solamente el trabajo de impacto mejora la DMO, el trabajo de fuerza sin impacto también ha sido un trabajo utilizado para la mejora de la DMO (Drinkwater et al., 1986; Hamdy et al., 1994; Lewis y Modlesky, 1998; Pocock et al., 1989). Existe diversidad en los resultados hallados en relación a la natación y la DMO. Hay una serie de estudios que indican que la natación puede ayudar a mejorar tanto la DMO como el SI con respecto al grupo que no entrena (Tsukahara; et al., 1994; Yurtkuran, 2005), mientras que otros indican lo contrario, que la natación no mejora la DMO con respecto al grupo que no realiza entrenamiento (Fehling et al., 1995; Heinrich et al., 1990; Harush y Rotstein, 2005).

La inactividad física se presenta como uno de los factores de riesgo más importantes a la hora de prevenir la aparición de la osteoporosis (Bloomfield, 2005), de ahí que sea necesario realizar al menos 30 minutos de actividad física moderada o intensa 5 días a la semana (Haskell et al., 2007).

Debido al tiempo necesario para encontrar cambios en la DMO, el objetivo de este artículo es evaluar el efecto de un programa de natación en piscina profunda y otro en piscina poco profunda sobre la mejora del SI en mujeres osteopénicas y postmenopáusicas. Además se pretende comprobar si alguno de estos programas de actividades acuáticas modifica los hábitos de actividad física en la vida cotidiana en mujeres osteopénicas y postmenopáusicas.

\section{Material y métodos}

Se evaluaron a 505 mujeres para medir el SI como variable de inclusión en el estudio. De éstas 505 mujeres, 177 presentaros valores que correspondían a osteopenia según la clasificación de la OMS (Kanis, 1994). Del total de participantes que tenían osteopenia fueron eliminadas aquellas que recibían algún tipo de tratamiento que no fuese calcio. Del total de las que cumplían todos los requisitos, se solicitó la participación de las mismas. Un total de 60 mujeres fueron incluidas en el estudio y divididas de forma aleatoria en dos grupos: 
Díaz, G.; Carrasco, M.; Barriga, A.; Jiménez, F.; Navarro, F. (2010). Efecto de dos programas de actividad física en el medio acuático con diferente impacto sobre el nivel de densidad ósea y el nivel de actividad física en mujeres postmenopáusicas y osteopénicas de Toledo. Revista Internacional de Ciencias del Deporte, 20(6), 196-204. http://www.cafyd.com/REVISTA/02002.pdf

1. Grupo de natación (GN), constaba de 30 participantes (edad=58,95 $\pm 7,05$ años; peso= $64,0 \pm 8,26 \mathrm{~kg}$; talla $=157,49 \pm 6,56 \mathrm{~cm})$. Realizaron actividades en la piscina profunda utilizando la resistencia ejercida por el agua ante el avance de las participantes como elemento para el desarrollo muscular. Las actividades consistían en la mejora de la técnica de nado así como de la mejora cardiovascular debido al nado continuo.

2. Grupo de impacto y resistencia (GIR), constaba de 30 mujeres (edad $=57,7 \pm 5,75$ años; peso $=64,45+9,37 \mathrm{~kg}$; talla $=155,51+6,41 \mathrm{~cm})$. Realizaron actividades en la piscina poco profunda del tipo: saltos, carreras, tracciones, etc... Las participantes de este grupo utilizaron elementos que aumentaban la resistencia en el agua tales como: hidroboots, minifins, aletas, palas, gomas, mancuernas de agua, etc.

El programa de entrenamiento consistió en 6 meses de tratamiento, dos sesiones por semana. Cada sesión tenía una duración de 45 minutos divididos en 10 minutos de calentamiento, 30 minutos de trabajo específico del grupo al que pertenecía y 5 minutos de vuelta a la calma. Este programa estaba diseñado y supervisado en todo momento por un licenciado en CC de la Actividad Física y el Deporte.

Las variables analizadas fueron:

o Peso y talla medidos con una báscula y tallímetro marca SECA con una precisión en el peso de $100 \mathrm{~g}$ y en la talla de $1 \mathrm{~mm}$.

o Nivel de actividad física, tanto en valores absolutos de intensidad contando los "counts" por hora, así como en el número de días que cumplen las recomendaciones diarias de 30 minutos de actividad moderada o vigorosa (MVPA). Para evaluar el nivel de actividad física de cada mujer, se utilizó un acelerómetro uniaxial MTI (Actigraph Manufacturing Technology, Inc). Este acelerómetro detecta aceleraciones verticales en un rango de 0,25 a $2,5 \mathrm{~Hz}$. Se colocó en la cintura durante 7 días analizando 4 de ellos, dos días de diario y el fin de semana. Se descartaron los dos primeros días y el último para evitar que las participantes cambiaran sus hábitos de actividad física por el hecho de llevar colocado el acelerómetro.

o Índice Cintura Cadera, entendida como la relación del perímetro de cintura con el perímetro de cadera. Para el cálculo de dicho índice se utilizó un cinta métrica Holtain de pvc con una precisión de $1 \mathrm{~mm}$.

o Para la evaluación de la DMO se utilizó el densitómetro portátil Lunar Aquilles Express, densitómetro de ultrasonidos aplicado en el talón. Se utilizó la T- Store del SI, valor que refleja el número de desviaciones típicas de la persona analizada con respecto a la media de la población joven, sana, de su misma raza y sexo. El SI se calcula a través de una fórmula en la que combina la atenuación del sonido (BUA) y la velocidad del sonido (SOS) (Njeh, et al., 1997).

Para el análisis estadístico se utilizó el paquete estadístico SPSS 12.0. Los valores medios al comienzo del estudio fueron analizados utilizando los estadísticos descriptivos de media y desviación típica. Una vez comprobada la normalidad y la homocedasticidad de la muestra, se evaluaron las diferencias entre ambos grupos mediante la realización de una prueba $\mathrm{T}$ para muestras independientes con el fin de comparar las medias intergrupo. Para analizar las diferencias intragrupo, realizamos una prueba $\mathrm{T}$ para muestras relacionadas. El nivel de significación aceptado para el análisis fue de $\mathrm{p}<0.05$ 
Díaz, G.; Carrasco, M.; Barriga, A.; Jiménez, F.; Navarro, F. (2010). Efecto de dos programas de actividad física en el medio acuático con diferente impacto sobre el nivel de densidad ósea y el nivel de actividad física en mujeres postmenopáusicas y osteopénicas de Toledo. Revista Internacional de Ciencias del Deporte, 20(6), 196-204. http://www.cafyd.com/REVISTA/02002.pdf

\section{Resultados}

Tras la división de los grupos se analizaron las diferencias entre los mismos al comienzo del estudio, observando que no había diferencias significativas en ninguna de las variables evaluadas entre ambos grupos tal y como se muestra en la tabla 1, por lo que los grupos son homogéneos antes de comenzar el estudio.

Tabla 1.- Variables descriptivas de ambos grupos en el momento PRE-

\begin{tabular}{|c|c|c|c|}
\hline Variables & $\begin{array}{c}\text { GIR } \\
(\mathrm{X} \pm \mathrm{DS})\end{array}$ & $\begin{array}{c}\mathrm{GN} \\
(\mathrm{X} \pm \mathrm{DS})\end{array}$ & $p$ \\
\hline Peso $(\mathrm{kg})$ & $66,76 \pm 15,23$ & $64,35 \pm 8,31$ & 0,105 \\
\hline Talla $(\mathrm{cm})$ & $155,9 \pm 6,39$ & $157,16 \pm 5,89$ & 0,411 \\
\hline ICC & $0,84 \pm 0,5$ & $0,85 \pm 0,05$ & 0,760 \\
\hline SI (TScore) & $-1,51 \pm 0,48$ & $-1,56 \pm 0,54$ & 0,998 \\
\hline $\begin{array}{c}\text { COUNTS } \\
\text { (Counts/hora) }\end{array}$ & $21527,34 \pm 9338,5$ & $22481,1 \pm 8706,42$ & 0,748 \\
\hline $\begin{array}{c}\text { MVPA } \\
\text { (días) }\end{array}$ & $2,07 \pm 1,44$ & $2,50 \pm 1,19$ & 0,351 \\
\hline
\end{tabular}

Tras los 6 meses de entrenamiento, los valores obtenidos por las participantes del estudio según el grupo al que pertenecían fueron los siguientes:

Tabla 2.- Variables descriptivas de ambos grupos en el momento POST-

\begin{tabular}{|c|c|c|c|}
\hline Variables & $\begin{array}{c}\mathrm{GIR} \\
(\mathrm{X} \pm \mathrm{DS})\end{array}$ & $\begin{array}{c}\mathrm{GN} \\
(\mathrm{X} \pm \mathrm{DS})\end{array}$ & $p$ \\
\hline Peso $(\mathrm{kg})$ & $66,24 \pm 15,02$ & $63,22 \pm 8,16$ & 0,37 \\
\hline ICC & $0,85 \pm 0,05$ & $0,84 \pm 0,04$ & 0,71 \\
\hline SI (TScore) & $-1,48 \pm 0,84$ & $-1,38 \pm 0,8$ & 0,66 \\
\hline $\begin{array}{c}\text { COUNTS } \\
\text { (Counts/hora) }\end{array}$ & $23253,91 \pm 8496,30$ & $23425,16 \pm 6561,42$ & 0,94 \\
\hline $\begin{array}{c}\text { MVPA } \\
\text { (días) }\end{array}$ & $2,53 \pm 1,12$ & $2,05 \pm 0,94$ & 0,17 \\
\hline
\end{tabular}

Tal y como se puede observar en los datos reflejados en la tabla 2, en el momento POST-, tras los 6 meses de evaluación, no hay diferencias significativas entre ambos grupos.

Con respecto a la evolución de cada una de las variables, a continuación se muestra en la figura 1 , la evolución en cada una de las variables. 
Díaz, G.; Carrasco, M.; Barriga, A.; Jiménez, F.; Navarro, F. (2010). Efecto de dos programas de actividad física en el medio acuático con diferente impacto sobre el nivel de densidad ósea y el nivel de actividad física en mujeres postmenopáusicas y osteopénicas de Toledo. Revista Internacional de Ciencias del Deporte, 20(6), 196-204. http://www.cafyd.com/REVISTA/02002.pdf

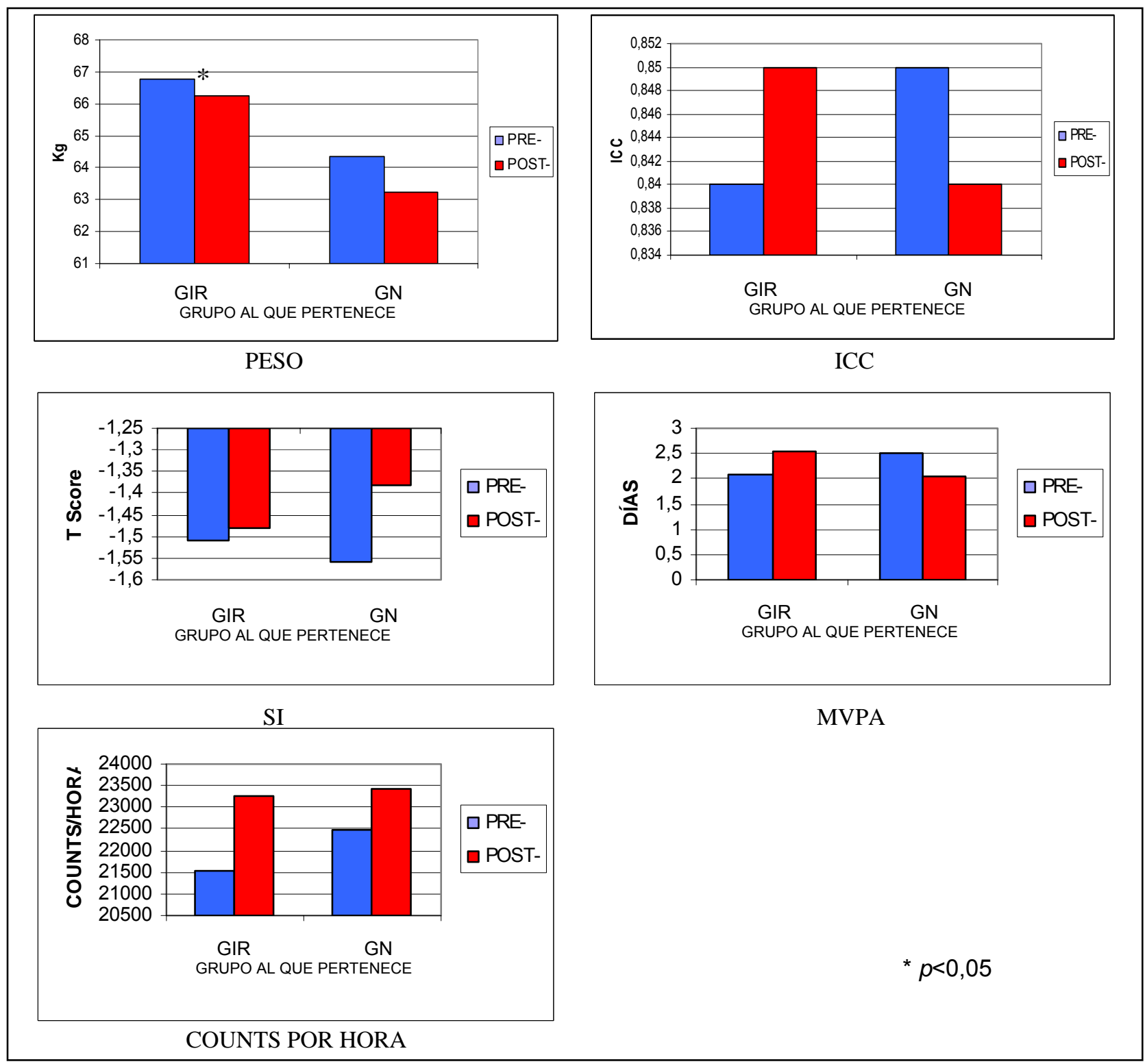

Figura 1.- Evolución de las diferentes variables

Como se puede observar en la figura 1 , sólo existen diferencias significativas $(p<0.05)$ entre el momento de evaluación PRE- y POST- en el grupo de piscina poco profunda en la variable del peso. En el resto de variables no encontramos diferencias ni entre ambos grupos, ni en cada grupo tras la finalización del tratamiento. 
Díaz, G.; Carrasco, M.; Barriga, A.; Jiménez, F.; Navarro, F. (2010). Efecto de dos programas de actividad física en el medio acuático con diferente impacto sobre el nivel de densidad ósea y el nivel de actividad física en mujeres postmenopáusicas y osteopénicas de Toledo. Revista Internacional de Ciencias del Deporte, 20(6), 196-204. http://www.cafyd.com/REVISTA/02002.pdf

\section{Discusión}

En el presente estudio se ha pretendido observar si alguno de los dos programas de actividad física en el medio acuático, con diferente impacto, tenían algún efecto sobre la calidad del hueso y, por consiguiente, en la reducción del riesgo de sufrir una fractura por osteoporosis.

En la bibliografía se puede observar que el ejercicio de impacto mejora el nivel de DMO en Bravo et al., 1996; Rutherford, 1999. Sin embargo, no es necesario que sean ejercicios de alto impacto, como saltos, para provocar una mejora de la DMO. Bastan ejercicios de bajo impacto, como andar o subir escaleras, para la mejora de la disminución de la DMO (Dalsky et al., 1988; Grove y Londeree, 1992; Taaffe et al., 1995).

El programa que se realizó en la piscina poco profunda intenta unir un trabajo de bajo impacto con un aumento del trabajo de fuerza con el uso de implementos para el medio acuático. Por estos motivos, se considera que este trabajo podría ser adecuado para la mejora de la DMO, aunque en el presente estudio no existan diferencias significativas.

Tras analizar los resultados obtenidos, se observó que dos días por semana durante seis meses de entrenamiento, no es suficiente estímulo para que la mejora del SI en el grupo de piscina poco profunda sea significativa, si bien existe una tendencia al aumento de este valor. Estos resultados coinciden con los encontrados por Harush y Rotstein (2005). Sin embargo difieren de los hallados por Ay y Yurtkuran, (2005) que sí encontraron mejoras significativas entre el GIR con respecto al grupo de control favorable al primero.

Con respecto al programa de natación en la piscina profunda, hay diversidad de opiniones sobre si un programa de natación puede (Orwoll et al., 1989; Pinto et al., 2003; Taaffe et al., 1995; Tsukahara et al., 1994) o no (Courteix et al., 1998; Fehling et al., 1995; Heinrich et al., 1990; Matsumoto et al., 1997) mejorar el nivel de DMO. No se han encontrado estudios en los que evalúen el SI tras un programa de entrenamiento en piscina profunda como el que se ha hecho en este estudio. En este estudio, se observó que el entrenamiento realizado no ha sido suficiente para producir un cambio significativo en el SI a pesar de que existe una tendencia al aumento del mismo.

Si se comparan los dos grupos, ambos grupos presentan un aumento en el SI pero no existen diferencias significativas entre ambos tras los 6 meses de entrenamiento, lo que no nos permite afirmar que un programa sea mejor que el otro para la mejora del SI. Sin embargo existe una tendencia a que los dos programas permitan recuperar SI tras 6 meses de entrenamiento.

Con respecto al nivel de actividad física realizada en su vida cotidiana, no se han podido encontrar estudios en los que se relacione el SI y el nivel de actividad física medido de forma directa. La mayoría de los estudios encontrados realizan la valoración de la actividad física mediante cuestionarios (Blanchet, et al., 2003; Landin-Wilhelmsen, et al., 2000). En este estudio, se encuentra una tendencia al aumento del nivel de actividad física en el grupo de piscina poco profunda, ya que aumenta tanto el número de "COUNTS"/hora como el número de días que cumplen las recomendaciones, pero ninguno de estos cambios son significativos. Sin embargo en el grupo de piscina profunda, aunque tiene una tendencia al aumento de los “COUNTS”/hora, presenta una tendencia a la disminución de los días que cumplen las recomendaciones sin ser ninguno de estos cambios, significativos. En consecuencia ninguno de los programas de actividad física en el medio acuático ha ayudado a aumentar la actividad física que realizan en su vida cotidiana de forma significativa. 
Díaz, G.; Carrasco, M.; Barriga, A.; Jiménez, F.; Navarro, F. (2010). Efecto de dos programas de actividad física en el medio acuático con diferente impacto sobre el nivel de densidad ósea y el nivel de actividad física en mujeres postmenopáusicas y osteopénicas de Toledo. Revista Internacional de Ciencias del Deporte, 20(6), 196-204. http://www.cafyd.com/REVISTA/02002.pdf

\section{Conclusión}

Una vez analizadas las diferentes variables para medir el efecto de dos programas de entrenamiento para la mejora de la DMO y el nivel de actividad física en mujeres postmenopáusicas y con osteopenia con una duración de seis meses, se puede concluir que:

o No existen cambios significativos a nivel del SI en ninguno de los dos grupos, aunque se produce una tendencia a la mejora del SI, más acentuada en el grupo de piscina profunda.

o A pesar de existir una tendencia al aumento de la actividad física cotidiana, tanto en el volumen como en la intensidad en el GIR y en el volumen en el GN, no se han producidos cambios significativos en el nivel de actividad física realizado por las participantes

\section{Referencias bibliográficas}

Asikainen, T.; Kukkonen-Harjula, K., y Miilumpalo, S. (2004). Exercise for Health for Early Postmenopausal Women. A systematic review of randomised controlled trials. Sports Medicine, 34(11), 753-778.

Astrom, J.; Ahnqvist, S.; Beertema, J., y Jonsson, B. (1987). Physical activity in women sustaining fracture of the neck of the femur. J Bone J oint Surg Br, 69(3), 381-383.

Ay, A y Yurtkuran, M. (2005). Influence of aquatic and weight-bearing exercises on Quantitative Ultrasound Variables in Postmenopausal. Am J Phys Med Rehabil, 84, 5261.

Bonaiuti, D.; Shea, B.; Iovine, R.; Negrini, S.; Robinson, V.: Kemper, H., et al. (2006). Ejercicios para la prevención y el tratamiento de la osteoporosis en mujeres postmenopáusicas (Revisión Cochrane traducida). La Biblioteca Cochrane Plus, 3, 129.

Blanchet, C., Giguère, Y., Prud'homme, D., Turcot-Lemay, L., Dumont, M., Leduc, G., et al. (2003). Leisure Physical Activity is Associated with Quantitative Ultrasound Measurements Independently of Bone Mineral Density in Postmenopausal Women. Calcified Tissue International, 73(4), 339-349.

Bloomfield, S. A. (2005). Contributions of physical activity to bone health over the lifespan. Topics in Geriatric Rehabilitation, 21(1), 68-76.

Bravo, G.; Gauthier, P.; Roy, P.; Payette, H.; Gaulin, P.; Harvey, M., et al. (1996). Impact of a 12-month exercise program on the physical and psychological health of osteopenic women. J Am Geriatr Soc, 44(7), 756-762.

Cannata, J.; Díez, A., y López, J. (2005). Estudio de coste-efectividad de protelos en al prevenciónm de fracturas osteoporóticas. Rev Esp Econ Salud, 4(3), 146-152.

Cooper, C.; Barker, D., y Wickham, C. (1988). Physical activity, muscle strength, and calcium intake in fracture of the proximal femur in Britain. BMJ, 297(6661), 14431446.

Coupland, C. A.; Cliffe, S. J.; Bassey, E. J.; Grainge, M. J.; Hosking, D. J., y Chilvers, C. E. (1999). Habitual physical activity and bone mineral density in postmenopausal women in England. International J ournal of Epidemiology, 28(2), 241- 246.

Courteix, D.; Lespessailles, E.; Peres, S.; Obert, P.; Germain, P., y Benhamou, C. (1998). Effect of physical training on bone mineral density in prepubertal girls: a comparative study between impact-loading and non-impact-loading sports. Osteoporosis International, 8(2), 152-158. 
Díaz, G.; Carrasco, M.; Barriga, A.; Jiménez, F.; Navarro, F. (2010). Efecto de dos programas de actividad física en el medio acuático con diferente impacto sobre el nivel de densidad ósea y el nivel de actividad física en mujeres postmenopáusicas y osteopénicas de Toledo. Revista Internacional de Ciencias del Deporte, 20(6), 196-204. http://www.cafyd.com/REVISTA/02002.pdf

Dalsky, G.; Stocke, K.; Ehsani, A.; Slatopolsky, E.; Lee, W., y Birge, S. J. (1988). Weight-bearing exercise training and lumbar bone mineral content in postmenopausal women. Ann Intern Med, 108(6), 824-828.

Drinkwater, B. L., y cols. (1995). Osteoporosis and Exercise. American College of Sports Medicine, 27, 1-7.

Drinkwater, B. L.; Nilson, K.; Ott, S., y Chesnut, C. H., 3rd. (1986). Bone mineral density after resumption of menses in amenorrheic athletes. JAMA, 256(3), 380-382.

Fehling, P. C.; Alekel, L.; Clasey, J.; Rector, A., y Stillman, R. J. (1995). A comparison of bone mineral densities among female athletes in impact loading and active loading sports. Bone, 17(3), 205-210.

Feskanich, D.; Willett, W., y Colditz, G. (2002). Walking and leisure-time activity and risk of hip fracture in postmenopausal women. JAMA, 288(18), 2300- 2306.

Grove, K., y Londeree, B. (1992). Bone density in postmenopausal women: high impact vs low impact exercise. Medicine and Science in Sports and Exercise, 24(11), 11901194.

Hamdy, R.; Anderson, J.; Whalen, K., y Harvill, L. (1994). Regional differences in bone density of young men involved in different exercises. Medicine and Science in Sports and Exercise, 26(7), 884-888.

Harush, D., y Rotstein, A. (2005). The effect of a water exercise program on bone density among postmenopausal women. AEA Aquatic Fitness Research Journal, 2(1).

Haskell, W. L.; Lee, I.M; Pate, R.R; Powell, K.E; Blair, S.N; Franklin, B.A; Macera, C.A; Heath, G.W; Thompson, P.D, y Bauman, A. (2007). Physical Activity and Public Health: Updated Recommendation for Adults from the American College of Sports Medicine and the American Heart Association. Medicine and Science in Sports and Exercise, 39(8), 1423-1434.

Heinrich, C.; Going, S.; Pamenter, R.; Perry, C.; Boyden, T., y Lohman, T. (1990). Bone mineral content of cyclically menstruating female resistance and endurance trained athletes. Medicine and Science in Sports and Exercise, 22(5), 558-563.

Kanis, J. (1994). Assessment of fracture risk and its application to screening for postmenopausal osteoporosis: synopsis of a WHO report. WHO Study Group. Osteoporosis International, Nov;4(6), 368-381.

Kanis, J. A., y Johnell, O. (2005). Requirements for DXA for the management of osteoporosis in Europe. Osteoporosis International: A Journal Established As Result Of Cooperation Between The European Foundation For Osteoporosis And The National Osteoporosis Foundation Of The USA, 16(3), 229-238.

Khan, K.; McKay, H.; Haapasalo, H.; Bennell, K.; Forwood, M.; Kannus, P., y cols. (2000). Does childhood and adolescence provide a unique opportunity for exercise to strengthen the skeleton? J Sci Med Sport, 3(2), 150-164.

Lewis, R., y Modlesky, C. (1998). Nutrition, physical activity, and bone health in women. International J ournal of Sport Nutrition, 8(3), 250-284.

Matsumoto, T.; Nakagawa, S.; Nishida, S., y Hirota, R. (1997). Bone density and bone metabolic markers in active collegiate athletes: findings in long-distance runners, judoists, and swimmers. International J ournal of Sport Medicine, 18(6), 408-412.

Njeh, C. F., Boivin, C. M., \& Langton, C. M. (1997). The role of ultrasound in the assessment of osteoporosis: A review. Osteoporosis International, 7(1), 7-22. 
Díaz, G.; Carrasco, M.; Barriga, A.; Jiménez, F.; Navarro, F. (2010). Efecto de dos programas de actividad física en el medio acuático con diferente impacto sobre el nivel de densidad ósea y el nivel de actividad física en mujeres postmenopáusicas y osteopénicas de Toledo. Revista Internacional de Ciencias del Deporte, 20(6), 196-204. http://www.cafyd.com/REVISTA/02002.pdf

Orwoll, E. S.; Ferar, J.; Oviatt, S. K.; McClung, M. R., y Huntington, K. (1989). The relationship of swimming exercise to bone mass in men and women. Arch Intern Med, 149(10), 2197-2200.

Pinto, A.; Takayama, L.; Carazzato, J.; Pereira, R., y Lima, F. (2003). The beneficial effect of swimming on bone mass. Medicine and Science in Sports and Exercise, 35(5), Suplement 1 S365.

Pocock, N.; Eisman, J.; Gwinn, T.; Sambrook, P.; Kelly, P.; Freund, J., y cols. (1989). Muscle strength, physical fitness, and weight but not age predict femoral neck bone mass. Journal of bone and mineral research, 4(3), 441-448.

Pruit, L.; Taaffe, D., y Marcus, R. (1995). Effects of a one-year high-intensity versus lowintensity resistance training program on bone mineral density in older women. Journal of bone and mineral research, 10(11), 1788-1795.

Rutherford, O. (1999). Is there a role for exercise in the prevention of osteoporotic fractures? Br J Sport Med, 33, 378-386.

Taaffe, D.; Snow-Harter, C.; Connolly, D.; Robinson, T.; Brown, M., y Marcus, R. (1995). Differential effects of swimming versus weight-bearing activity on bone mineral status of eumenorrheic athletes. Journal of bone and mineral research, 10(4), 586-593.

Tsukahara, N.; Toda, A.; Goto, J., y Ezawa, I. (1994). Cross-sectional and longitudinal studies on the effect of water exercise in controlling bone loss in Japanese postmenopausal women. J Nutr Sci Vitaminol, 40(1), 37-47.

Valdivia, G., y Szot, J. (1999). Epidemiología de la osteoporosis. Boletín de la escuela de medicina, 28(1-2), 1-8.

Wickham, C.; Walsh, K.; Cooper, C.; Barker, D.; Margetts, B.; Morris, J., y cols. (1989). Dietary calcium, physical activity, and risk of hip fracture: a prospective study. BMJ , 299(6704), 889-892. 